\title{
ANALYSIS OF METHODS USING FOR SUPERRESOLUTION OF THE SIGNALS IN THE TASKS OF SPACE-TIME ACCESS
}

\author{
Mykola Moskalets \\ Department of Telecommunication Systems \\ Kharkiv National University of Radioelectronics \\ 14 Nauky ave., Kharkiv, Ukraine, 61166 \\ mykola.moskalets@nure.ua \\ Svitlana Teplytska \\ Department of Telecommunication Systems \\ Kharkiv National University of Radioelectronics \\ 14 Nauky ave., Kharkiv, Ukraine, 61166 \\ svitlana.teplytska@nure.ua
}

\begin{abstract}
Six methods are considered in the analysis of the methods of angular superresolution of the signals: non-adaptive beamforming, Kapon, thermal noise, Bordzhotti-Lagunas, maximal entropy and multiple signal classification (MUSIC). The comparative characteristic of the methods with assessing their advantages disadvantages and limitations is given. Theoretical resolution of these methods is assessed in the article.

Numerical evaluation of resolution ability of the methods of angular superresolution of the signal are obtained based on the simulation of various scenarios of signal-to-noise ratio, taking into account the use of correlated and uncorrelated signals, a different number of antenna elements and the values of the signal/interference+noise ratio. These estimations show the ultimate theoretical accuracy of the methods and the potential for their use in problems of space-time access with set limits.

The simulation results confirmed the statistical consistency of these methods of estimation of arrival direction angles of correlated and uncorrelated signals from subscriber stations for the space-time sampling in the output of the linear equidistant antenna array.

The present analysis and research results make it possible to select the most effective method for determining the arrival of signals in accordance with the given parameters of signal-to-noise ratio under restrictions.
\end{abstract}

Keywords: space-time access, angular superresolution, antenna array, direction of signal arrival.

\section{Introduction}

Currently, in the world and in Ukraine, there is shortage of using the frequency, time and code resources and there is a necessary for additional physical resources, implementing more efficient use of radio frequency resources, increase of network bandwidth and increase of the productivity of mobile communication system as a whole [1-3]. In this aspect, the use of promising methods space-time access (STA) methods in a mobile communication system is prospective [4].

The main issues in the STA problems using adaptive antenna arrays (AA) is the problem of determining the direction of arrival of signals from the mobile subscriber station (SS). This problem is solved on the basis of different methods: using an equisignal zone direction finding, estimations of AA directional characteristics, with the Rayleigh resolution, with superresolution (SR), etc. that have their performance $[5,6]$.

Methods for Direction of arrival estimation (DOA) are the basis of many telecommunications applications. Analysis of these methods in terms of their effectiveness and feasibility is sufficiently urgent task for solving STA problems in mobile communication systems.

The problem of determining the direction of arrival of the electromagnetic wave is as an independent value (radio emission sources direction finding) and an integral component of the more general problems of radiocommunication, radiolocation, navigation and other [7].

Estimation of the theoretical resolution ability of some superresolution methods are conducted by the author as a part of investigated problem. The values of ultimate theoretical accuracy of these methods are obtained based on the simulation of various scenarios of signal-to noise ratio. 


\section{Materials and methods}

Implementing the superresolution methods in the space domain, it is possible to determine the position of the spectral components (directions of arrival), analyzed (received) signals. In the works of Johnson and Kapon et al. [8, 9], methods of superresolution of the signals separated by an arbitrarily small distance were developed based on the methods of nonlinear spectral analysis.

There are a large number of algorithms realizing SR method. Among these are: [5]:

1) methods based on the determination of the local maxima positions of pseudospectrum $\mathrm{D}(\theta)$ at the space scanning: Kapon, "thermal noise" and Bordzhotti-Lagunas;

2) methods based on the spatial correlation matrix R decomposition into the eigenvectors $\mathrm{Vk}$ and the $\mathrm{k}$ - eigenvalues - MUSIC method, EV (eigenvector);

3) methods based on the separation of signal and noise spaces. There are ROOT-MUSIC and ESPRIT methods, focused on the use of linear equivalent AA.

Generalized comparative characteristics of the most popular methods to evaluate the direction of the angular superresolution of the signal is shown in Table $\mathbf{1 .}$

It is interest to obtain the resolution estimation of the presented methods of angular superresolution depending on the number of arrival signals from different directions, the values of the signal/(noise+interference) ratio (SNR), signal correlations and their quantity, the number of antenna array elements based on the analysis of the Table 1.

\section{Research of estimation methods of the arrival direction of the signals with superresolution}

Investigation of estimation methods of the arrival direction of the signals with superresolution of 8-element equidistant linear AA is conducted on the basis of simulation in MatLab13.h package.

The basis of the analysis of superresolution methods is the next: non-adaptive beamforming (NBF), Kapon, thermal noise, Bordzhotti-Lagunas, maximum entropy (MEM), multiple signal classification (MUSIC).

Resolution estimation of the methods of angular superresolution of the signals by the proposed method is based on simulation of 5 signal-to-noise ratio scenarios with variations of different parameters.

Values of parameters, which close to the limit of stability of methods, were used in order to obtain adequate quantitative and qualitative assessments of the theoretical limit of accuracy of methods and presented in the Table 2.

The aim of the task of evaluation of angular superresolution methods of the signals is to determine the angular coordinates of radiation sources under observation of M-data sample from outputs of 8 antenna elements of equidistant linear AA and comparative analysis of the methods according to the resolution criterion.

We use a number of assumptions in this study:

- the number of estimated signals is known (this allows to select the number of training samples equal to the optimum and thus avoid additional losses);

- the number of estimated signals does not exceed the number of antenna elements (otherwise, we obtain degenerative estimations);

- the signals are unknown deterministic functions (it is typical for communication systems);

- set of $\mathrm{N}$ any directed vectors is linearly independent (AA lattice set is unique just in case if any set of unique direction vectors $\mathrm{N}$ that form this lattice set in a multiply domain forms a linearly independent set at, where $\mathrm{M}$ - the AE number) [10];

- gaussian white noise is used as a noise in the processing band. It is uncorrelated in all spatial channels with the same capacity (additional evaluation of the noise parameters considerably complicates the task of estimating the direction of arrival of signals).

The analytical model for estimating the AA weight vector is much more complicated in the case of non-accounting of the assumptions due to the increase in the number of parameters defining the a priori information about observed AA processes. This significantly increases the computational complexity that makes the data model practically unrealizable. 
Table 1

Comparative characteristics of the evaluation methods of the arrival direction of angular superresolution signals

\section{Evaluation method of
arrival direction of the signals}

1 The classical method of beamforming

(Bartlett's method or the method of delays - the expansion of the spectral Fourier analysis), [10]:

$D_{F}(\theta)=\frac{\vec{a}(\theta)^{H} R_{x x} \vec{a}(\theta)}{\vec{a}^{H}(\theta) \vec{a}(\theta)}$,

$\vec{v}(\theta)$ - AA response vector, i. e. columns of weight coefficients for all angles of arrival of signals

2 Algorithms for linear prediction (autoregressive model algorithm or maximum entropy method (MEM)), [11]:

$$
\mathrm{D}_{\text {MEM }}(\theta)=\mathrm{R}_{11}^{-1} /\left|\overrightarrow{\mathrm{a}}(\theta)^{\mathrm{H}} \mathrm{R}_{1}^{-1}\right|^{2} \text {. }
$$

Application possiirregular structure of the antenna components (AS)

The applicability to the analysis of time series (time range). Restricting the use of a spatial spectrum estimation of linear equidistant lattice with a uniform amplitude distribution

3 Kapon method (Minimum variance method (MVDL) - the method of minimal deviation o the minimal variation (dispersion)), [9]: $\mathrm{D}_{c}(\theta)=1 /\left(\overrightarrow{\mathrm{a}}(\theta)^{\mathrm{H}} \mathrm{R}^{-1} \overrightarrow{\mathrm{a}}(\theta)\right)$.

4 Thermal Noise Algorithm (TNA), [12]: $\mathrm{D}_{\mathrm{TNA}}(\theta)=1 /(\overrightarrow{\mathrm{a}}(\theta) \quad \mathrm{R} \quad \overrightarrow{\mathrm{a}}(\theta))$.

A high-resolution algorithm is obtained using high degrees of inverse correlation matrix: $\mathrm{D}(\theta)=1 /(\overrightarrow{\mathrm{a}}(\theta) \quad \mathrm{R} \quad \overrightarrow{\mathrm{a}}(\theta)), \mathrm{k}>2$

5 Borjotti-Lagunas method [13]:

$$
D_{B L}(\theta)=\frac{D_{C}}{D_{T N A}}=\frac{\vec{a}(\theta)^{H} R^{-1} \vec{a}(\theta)}{\vec{a}(\theta)^{H} R^{-2} \vec{a}(\theta)} .
$$

6 MUSIC method

(Multiple signal classification), [14]:

$$
\mathrm{D}_{\text {Music }}(\theta)=\frac{1}{\overrightarrow{\mathrm{a}}(\theta)^{\mathrm{H}}\left(\sum_{\mathrm{k}=\mathrm{M}+1}^{\mathrm{R}} \mathrm{Q}_{\mathrm{k}} \cdot \mathrm{Q}_{\mathrm{k}}^{\mathrm{H}}\right) \overrightarrow{\mathrm{a}}(\theta)},
$$

where $Q_{k}$ - eigenvectors of the correlation matrix R; $\mathrm{M}$ - the number of radiation sources

Apply to any AA configuration (one-, two-, three-dimensional, regular and irregular AA) in comparison with the Fourier method

Resolving power increases under ideal conditions (excluding technical performance and hardware the Kapon method It refers to a group of algorithms based on mal noise parameters

Large resolution in comparison with the Kapon method. The method is effective when the signal/noise ratio and the number of bility of AA with an

A simple implemen-
tation

Advantages

Restrictions and disadvantages of the method

The method is based on the AA beam scanning in the space and determining the maximum level of radiation. Low resolution is determined by the width of the main AA beam and minor lobe levels Resolution depends on the width of the main AA beam and is connected with the restriction of Rayleigh resolution.

The disadvantage can be reduced by increasing the number of AA elements.

The increase in $\mathrm{S} / \mathrm{N}$ ratio or increase in observation time does not change the resolution

The complexity of the correct evaluation of the required order of autoregressive model - filter length (vector length $\mathrm{R}_{11}^{-1}$ ).

Spectrum is smoothed at a small filter length, but with poor resolution and very large length of prediction filter, with good resolution, resulting in highly fluctuating range - a high level of minor lobes of a random nature. Increase of resolution errors) compared with evaluation of the ther-

AA elements tend to infinity.

It is apply to any AA configuration
The necessity for a large number of training samples (twice the number of lattice elements) that increases the volume of computational operations.

Low efficiency of the method with small sample sizes and the initial data.

Inability of signal detecting from strongly correlated sources.

The number of AA elements does not impose restrictions on the resolution. Resolution grows unrestricted by increasing the signal/noise ratio and the sample size Real resolution remains the same with increasing $\mathrm{k}$ degree, but the computational complexity significantly increases

Normalization of the signal/noise ratio at the AA output by the thermal noise power

MUSIC method is the limiting case of Kapon when $\mathrm{S} / \mathrm{N}$ ratio tends to infinity.

The necessity for a preliminary evaluation of the dimension of the signal and noise subspaces.

It is critical to the uncertainties of the parameters of the mathematical model and to the value of signal/noise ratio, when the sources are closely spaced.

High computational complexity of the method is determined by calculating the singular decomposition of the covariance matrix of outputs of AA elements.

The method does not give results for correlated signal sources. Root-MUSIC allows to find the coherent signals 
Table 2

Initial data for the implementation of simulation scenarios of the valuation methods of arrival directions of the angular superresolution signals

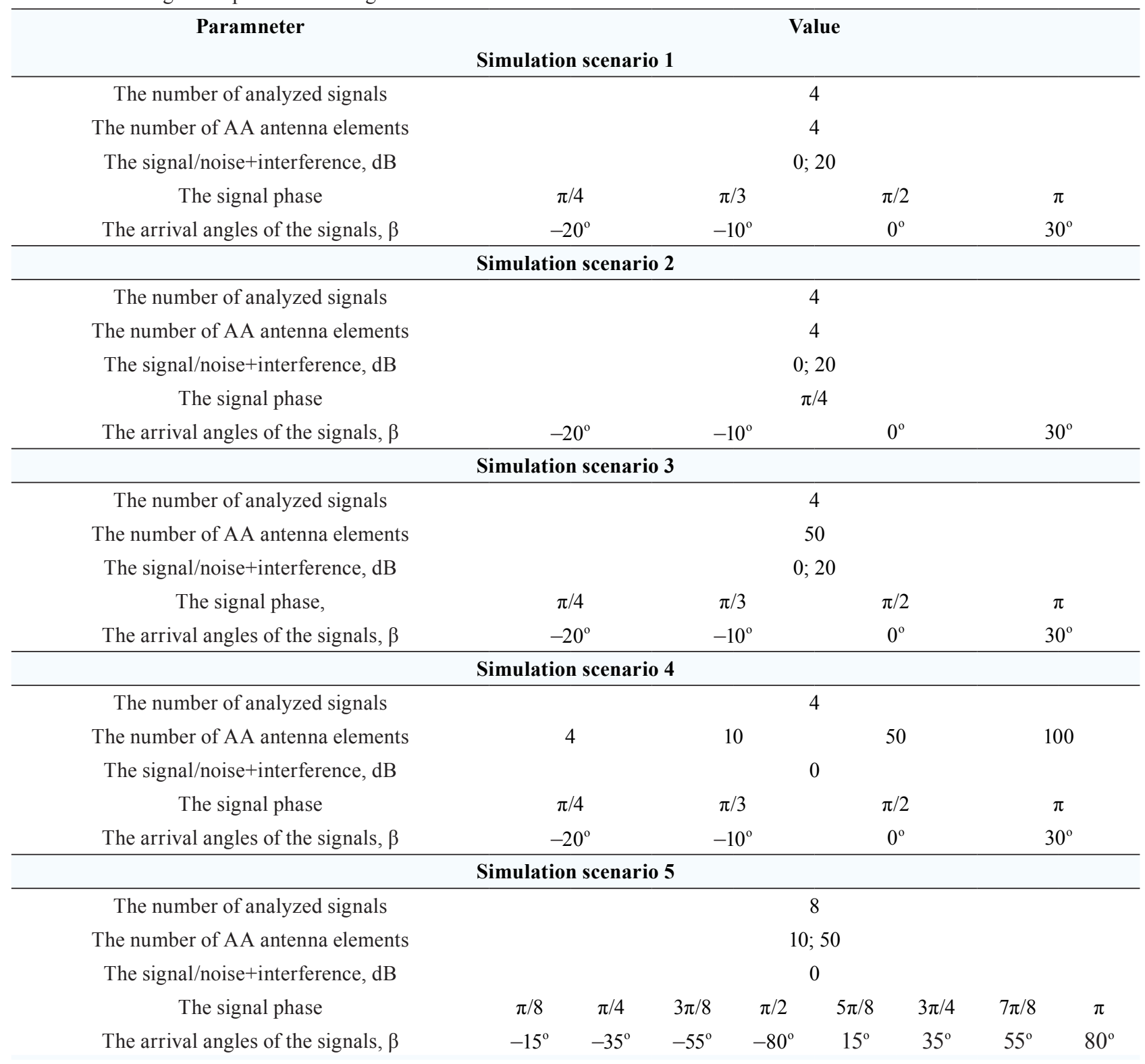

\section{The simulation results of the resolution evaluation of angular superresolution methods}

Scenario 1. Uncorrelated signals are considered from the 4 point radiation sources with the arrival angles: $-20^{\circ},-10^{\circ}, 0^{\circ}, 30^{\circ}$ and phase $\pi / 4, \pi / 3, \pi / 2$ and $\pi$ (Table 2). The number of AA elements -10 , the distance between the $\mathrm{AE}-\mathrm{d}=\lambda / 2$. Signal/noise ratio: $0.20 \mathrm{~dB}$. Analysis of the curves in Fig. 1 shows that a non-adaptive beamforming method (Fourier) does not allow the spatial separation of the signals, as can be seen 3 of the 4 signals are in the same resolution element. Other methods have higher resolution characteristics and allow to estimate the arrival angles of signals with different data accuracy. As can be seen, the most precision method is the MEM, at the same time the false peaks are observed in the spectrum. Only MUSIC and MEM methods provide a maximum capacity of arrival of signals from consideration areas. (Fig. 1, b) The resolution of the methods is significantly increased with the increase of SNR up to $20 \mathrm{~dB}$ except Bordzhotti-Lagunas and classical NAB methods. 

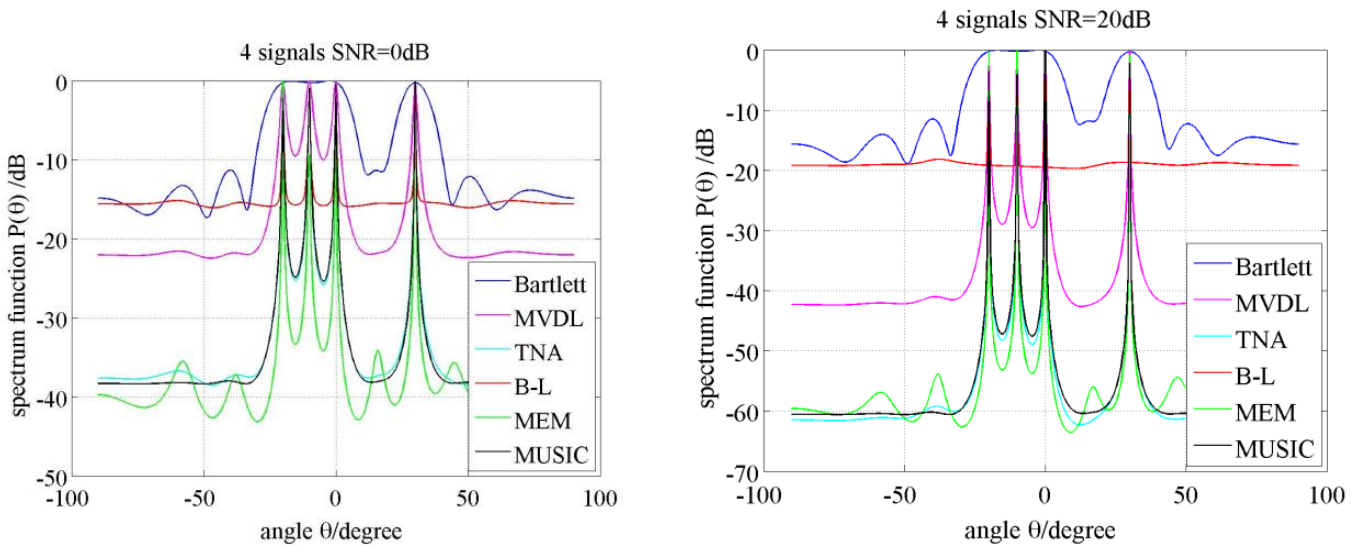

a

b

Fig. 1. The dependency graphs of emission power on the arrival angle of uncorrelated signals of superresolution methods: $a-\mathrm{SNR}=0 \mathrm{~dB} ; b-\mathrm{SNR}=20 \mathrm{~dB}$

Scenario 2. This scenario provides arrival of 4 correlated signals with the phase $\pi / 4$. As seen from the results shown in Fig. 2, $\boldsymbol{b}$ almost all methods aren't appropriate to the task of evaluation of the spatial separation of the radiation source at SNR ratio $0 \mathrm{~dB}$. The best result is provided by MEM method in the case of increase of SNR ratio up to $20 \mathrm{~dB}$.

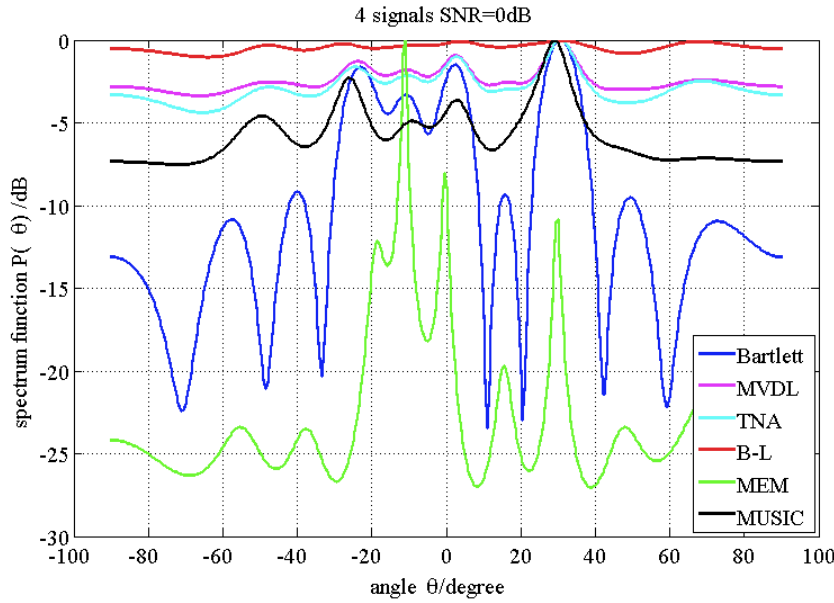

a

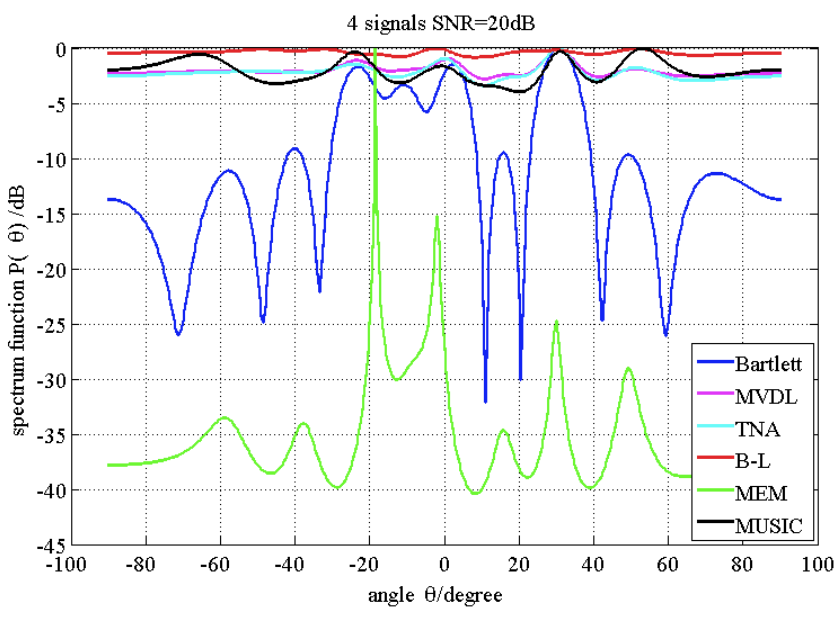

$b$

Fig. 2. The dependency graphs of emission power on the arrival angle of correlated signals of superresolution methods: $a-\mathrm{SNR}=0 \mathrm{~dB} ; b-\mathrm{SNR}=20 \mathrm{~dB}$ 
Scenario 3. Let's consider the option of AA use with the number of elements is 50 (Fig. 3, a). As can be seen from the analysis of the obtained relationships, MEM method gives false peaks for evaluation of spatial radiation of the sources, which generally degrades its characteristics regardless of the ratio (Fig. 3, b). The most accurate methods are MUSIC and Kapon methods. Reducing or increasing of the SNR ratio does not significantly affect the resolution of the method.

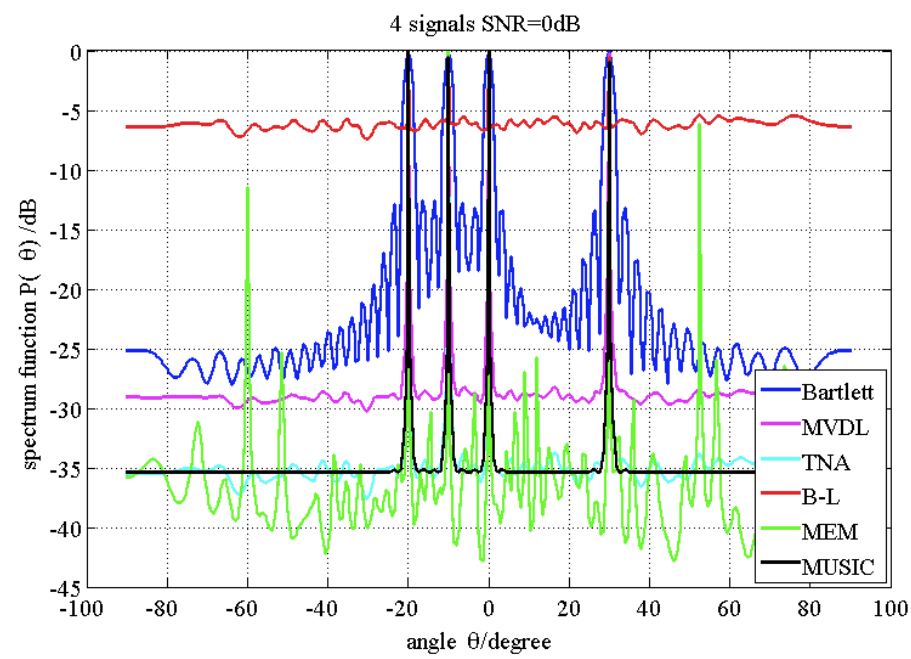

$a$

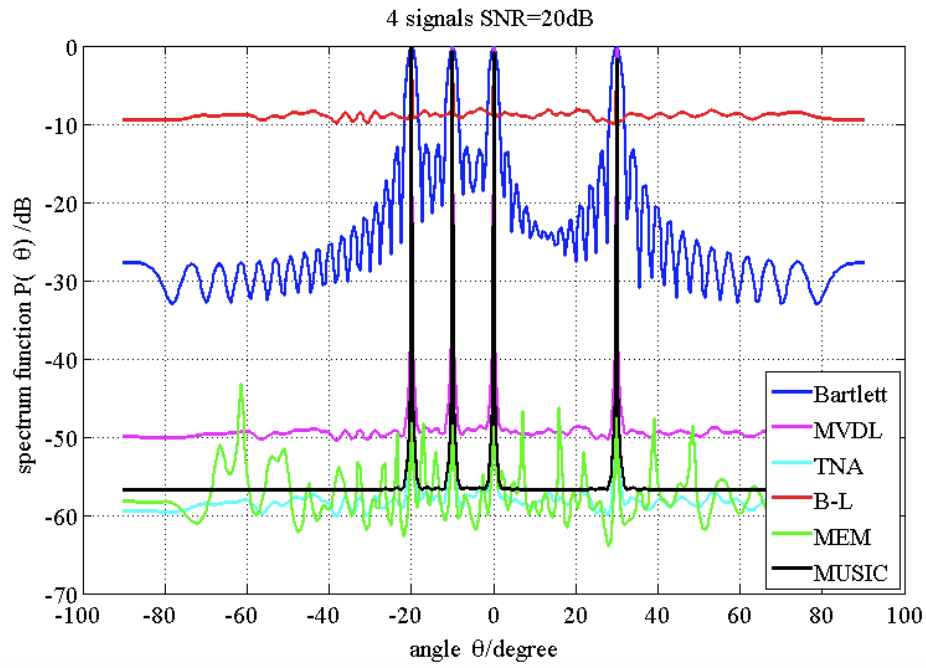

$b$

Fig. 3. The dependency graphs of emission power on the arrival angle of uncorrelated signals of superresolution methods with the number of AA elements: $\mathrm{N}=50: a-\mathrm{SNR}=0 \mathrm{~dB} ; b-\mathrm{SNR}=20 \mathrm{~dB}$

Scenario 4. Let's analyze the resolution of superresolution methods using AA with the number of AE: 4; 10; 50; 100 at SNR $=0 \mathrm{~dB}($ Fig. $4, \boldsymbol{a}-\boldsymbol{f})$. The nature of relations shows that the resolution of the direction evaluation of arrival of the signals for each of these methods can be significantly increased by increasing the number of AE.

Scenario 5. The estimation results of arrival direction for 8 signals with different phases and angles of arrival using the AA with the 10th and 50th AE are shown in Fig. 5, $\boldsymbol{a}-\boldsymbol{c}$ (Table 2). As can be seen from the nature of the dependencies in Fig. 5, $\boldsymbol{a}, \boldsymbol{b}$, the key parameter influencing the resolution is the ratio of the estimated number of signals and $\mathrm{AE}$.

As can be seen from the analysis of the dependencies in Fig. 5, that correlation matrix degenerates when the number of antenna elements is equal to 10 and neither of the methods provides the spatial separation of the signals, because the number of signals exceeds the sum of the average 
number of antenna elements and the rank of the correlation matrix $N>\frac{N+\operatorname{rank}\left\{R_{x x}\right\}}{2}[12]$.

Several improvement of spatial estimation result of arrival of signals is possible by increasing the number of AE to 50 items and as can be seen in Fig. 5, $\boldsymbol{b}$, consistently good results gives MUSIC method at values $\mathrm{SNR}=0 \mathrm{~dB}$.

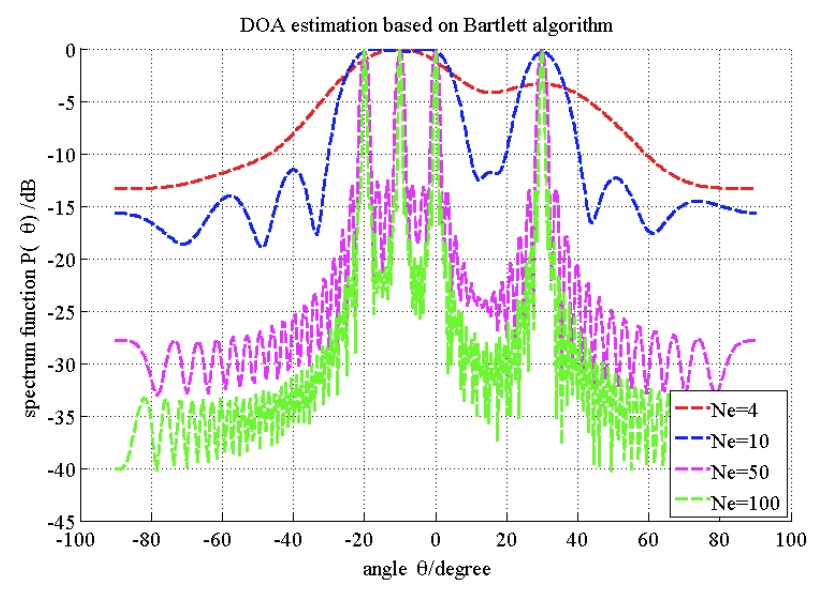

a

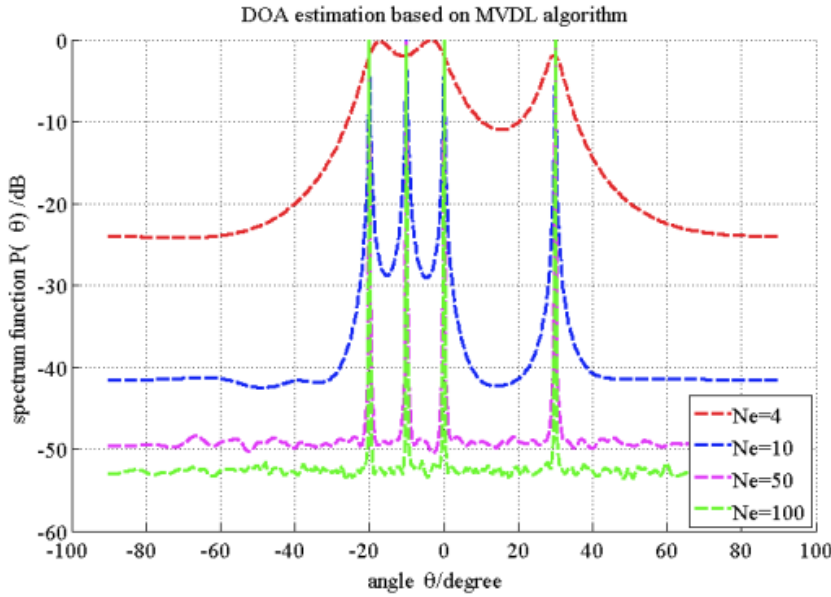

c

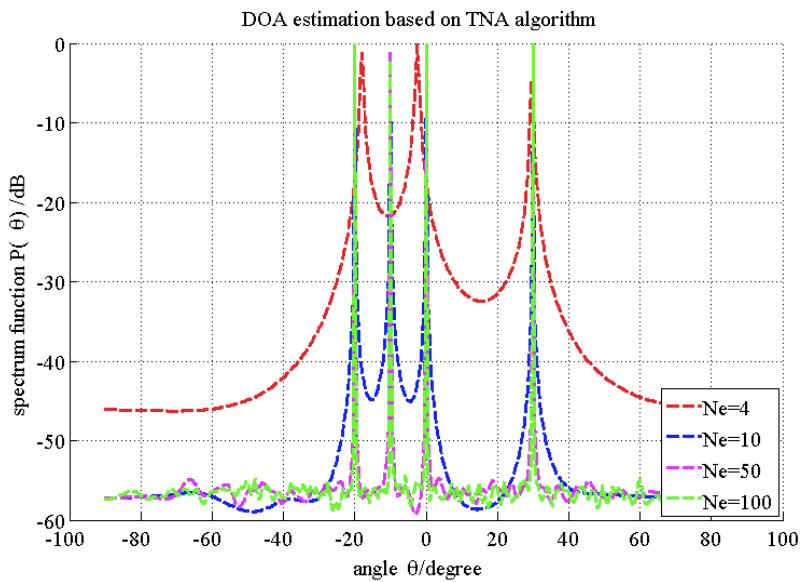

$e$

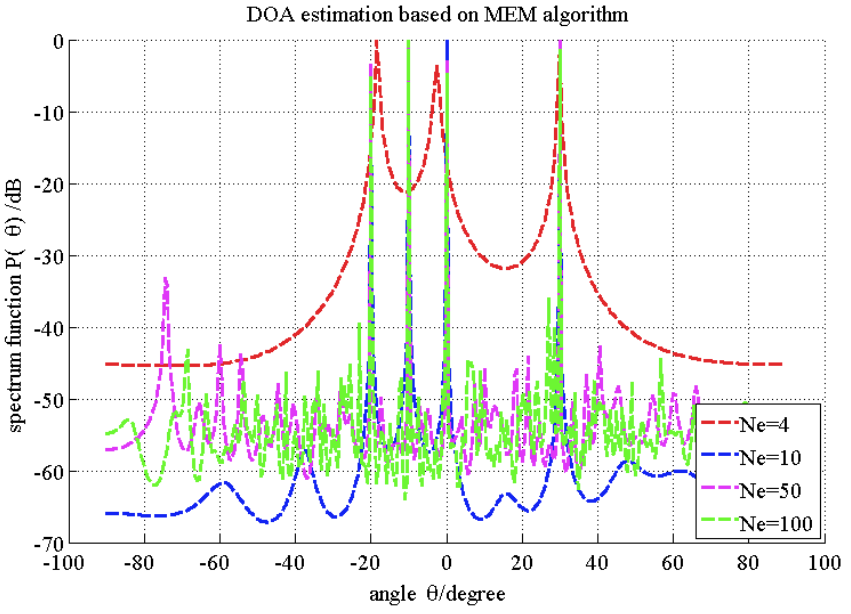

$b$

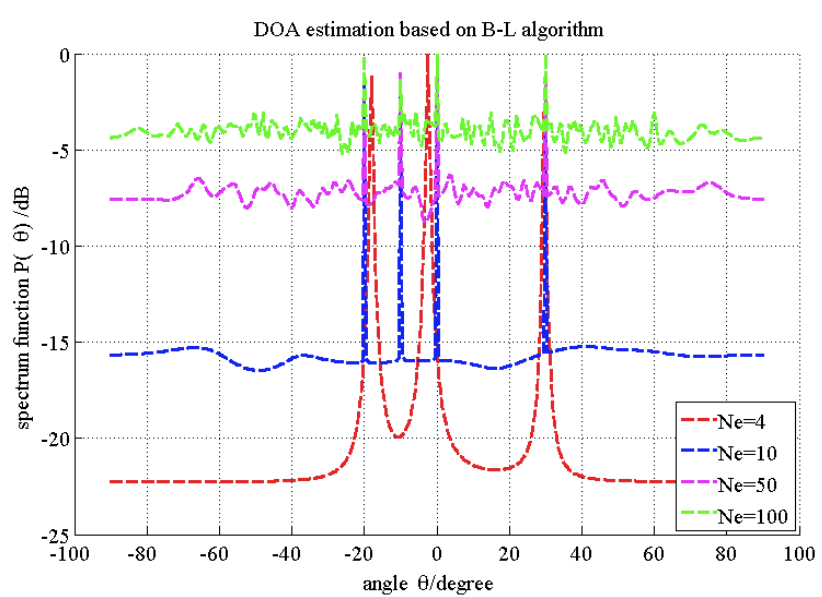

d

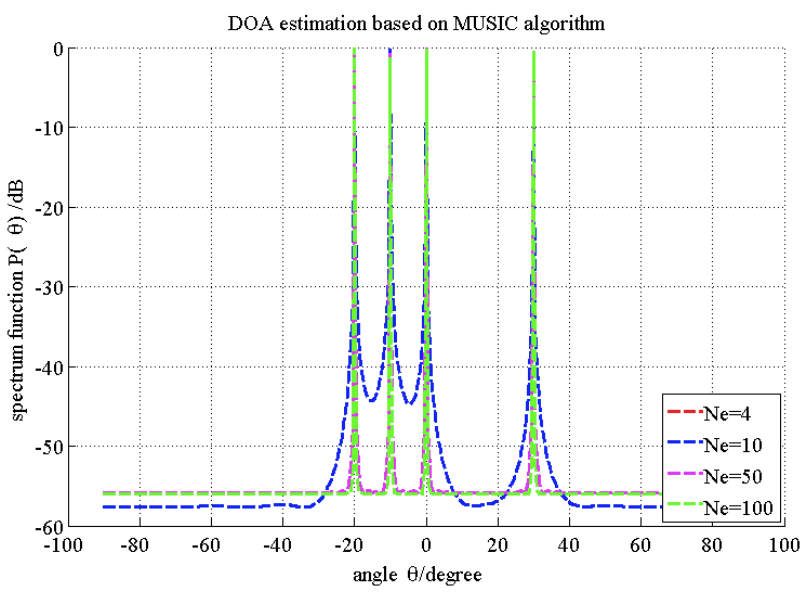

$f$

Fig. 4. The dependency graphs of emission power on the arrival angle of uncorrelated signals of superresolution methods with the number of AA elements $a-f: \mathrm{N}=4 ; 10 ; 50 ; 100$ and $\mathrm{SNR}=0 \mathrm{~dB}$ 


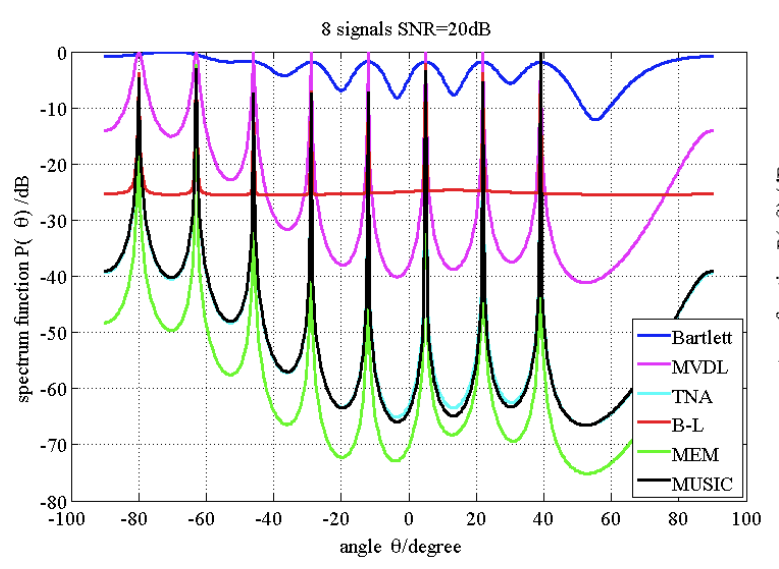

$a$

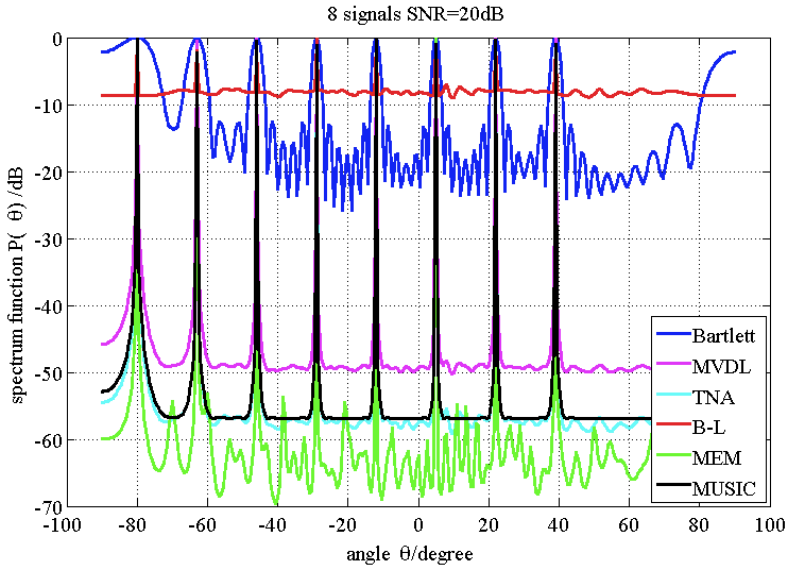

b

Fig. 5. The dependency graphs of emission power on the arrival angle of uncorrelated signals of superresolution methods with the number of AA elements: $a-\mathrm{N}=10 ; b-\mathrm{N}=50$ and $\mathrm{SNR}=0 \mathrm{~dB}$

It should be noted that the obtained estimation results of resolution of these methods are potentially achievable under these assumptions of analysis technique.

\section{Conclusions}

Analysis of the simulation results of resolution estimation of the arrival angles of the signals from 4 sources of radiation is shown:

1. Classic non-adaptive beamforming method is the easiest in the practice, however, it has a major disadvantage associated with the restriction of the Rayleigh resolution [9]. Analysis of curves in Fig. 4 is shown that this disadvantage can be decreased by the increase in the number of AE. Improvement of $\mathrm{S} / \mathrm{N}$ ratio or an increase in observation time does not change the resolution (Fig. 1, $\boldsymbol{a}, \boldsymbol{b}$ ).

2. Estimation of the spatial resolution of the sources using Kapon method exceeds the classical beamforming method, because it uses every available degree of freedom for the concentration of produced energy in the direction of interest bearing. Existing energy minimization power can be interpreted as a limitation of the ability to suppress noise for more focused zero formation in the direction of presence of other sources. This reduces the spectral dispersion with closely spaced sources, although the resolution of the antenna pattern forming depends on the signal-to-noise ratio according to Kapon (Fig. 1, a, b) and the SS aperture (Fig. 4, c).

3. Kapon method has the lowest resolution (Fig. 1, a) among the non-parametric methods of continuous analysis: "thermal noise" and Bordzhotti-Lagunas. However, when the AE number increases, which increases the final length of the sample on the background of random amplitude and phase errors in the receiving channels, Bordzhotti-Lagunas and "thermal noise" methods may lose the advantage compared with Kapon algorithm (Fig. 3, b).

4. The method of multi-signal classification MUSIC is a limiting case of Kapon method for signal/noise ratio tended to infinity, which is consistent with a higher resolution of MUSIC compared with the Kapon algorithm. Analyzing the graphs in Fig. 1, $\boldsymbol{a}, \boldsymbol{b}$, we conclude that the MUSIC method is able to provide a solution, regardless of the signal-to-noise ratio, the incoming signal at a sufficient angular separation of sources. However, from the analysis of $[6,9,11]$ it is known that the MUSIC method is critical to the uncertainties of the parameters of the mathematical model, as well as to the value of signal/noise ratio in the case where the sources have similar bearings. As seen from the graphs in Fig. 2, $\boldsymbol{a}, \boldsymbol{b}$, MUSIC method assumes uncorrelatedness of signal sources that in the real cases of multipath emission is not always feasible. If SNR value and time for data acquisition are sufficiently large and signal model is fairly accurate, the MUSIC method gives the statistically consistent estimations, surpassing other estimation methods discussed in the article (Fig. 1, b).

5. The simulation results confirm the statistical consistency of considered estimation methods of arrival angles of the signals from subscriber stations. Evaluation was carried out based on the spacetime in the output of 8-element equidistant linear array, considering the cross-correlation of the signals. 
6. Under ideal conditions, these superresolution methods make it possible to obtain the accurate values of the arrival directions of the signals. However, it is assumed that a higher quality of reception (signal/noise ratio) is necessary for the successful solution of the superresolution problem. Geometric and electromagnetic parameters of the AA, the exact coordinates of the AE installation will be precisely known, taking into account the condition $\mathrm{d}<\frac{\lambda}{2 \sin \theta_{\max }}$, where $\mathrm{d}-$ the distance between adjacent AA, parameters of resolved signals.

7. Based on the study, it becomes clear that the globally optimal superresolution method does not exist. The attractiveness of various methods is largely dependent on the presence and volume of a priori information about investigated processes, the method of evaluation of AA response vectors and its method of its use in relation to the current signal-to-noise ratio, AA configuration, its geometrical parameters and other.

In addition, there are some negative outcomes of decisions - the occurrence of false marks the detection of non-existing radiation sources, bias in the estimates of the arrival directions of signals.

The comparative analysis showed that the problem of using superresolution methods in the tasks of space-time access remains relevant for research, because the issues related to obtaining objective estimations of extreme accuracy of arrival angles of the signals depending on a number of individual parameters in the current signal-to-noise ratio are insufficiently studied.

The simulation results of methods with obtaining numerical estimations of resolution showed that the resolution of these methods allows their use in the tasks of space-time access under strictly limited circumstances.

\section{References}

[1] The METIS 2020 Project. Mobile and wireless communications Enablers for the 2020 Information. Society. EU FP7 ICT-317669-METIS Available at: https://www.metis2020.com/

[2] Hardouin, E. (2013). 5G: an operator's perspective. Orange Labs. LTE World Summit, 10.

[3] Avdyeyenko, K. L., Gepko, I. O., Korsak, V. F. (2015). Klyuchovi pry’ncy’py` spil’nogo dostupu do radiochastotnogo resursu na licenzijnij osnovi (LSA) “Zv'yazok”, 6, 49-52.

[4] Popovskij, ,V. V., Vasilenko, Ju. A. (2012). Jeffektivnoe ispol'zovanie vsego fizicheskogo prostranstva signalov v mobil'nyh telekommunikacionnyh sistemah. Mezhregional'nyj forum MSJe. Nacional'naja komissija, osushhestvljajushhaja gosudarstvennoe regulirovanie v sfere svjazi i informatizacii (NKRSI), 42-45.

[5] Classical and Modern Direction-of-Arrival Estimation (2009). Elsevierб 451. doi: 10.1016/ c2009-0-19135-3

[6] Chandran, S. (2006). Advances in Direction-of-Arrival Estimation. USA: Artech House, 474.

[7] Tarokh, V. (Ed.) (2009). New Directions in Wireless Communications Research. 483. doi: 10.1007/978-1-4419-0673-1

[8] Johnson, R. L., Miner, G. E. (1986). Comparison of Superresolution Algorithms for Radio Direction Finding. IEEE Transactions on Aerospace and Electronic Systems, AES-22 (4), 432-442. doi: 10.1109/ taes.1986.310779

[9] Capon, J. (1969). High-resolution frequency-wavenumber spectrum analysis. Proceedings of the IEEE, 57 (8), 1408-1418. doi: 10.1109/proc.1969.7278

[10] Monzingo, R. A., Miller, T. W. (1986). Adaptive antenna arrays: Introduction to the theory. Moscow: Radio and Communications, 486.

[11] Nickel, U. (1987). Angular superresolution with phased array radar: a review of algorithms and operational constraints. IEE Proceedings F Communications, Radar and Signal Processing, 134 (1), 53-59. doi: 10.1049/ip-f-1.1987.0010

[12] Gabriel, W. F. (1980). Spectral analysis and adaptive array superresolution techniques. Proceedings of the IEEE, 68 (6), 654-666. doi: 10.1109/proc.1980.11719

[13] Borgiotti, G., Kaplan, L. (1979). Superresolution of uncorrelated interference sources by using adaptive array techniques. IEEE Transactions on Antennas and Propagation, 27 (6), 842-845. doi:10.1109/ tap.1979.1142176

[14] Lavate, T. B., Kokate, V. K., Sapkal, A. M. (2010). Performance Analysis of MUSIC and ESPRIT DOA Estimation Algorithms for Adaptive Array Smart Antenna in Mobile Communication. International Journal of Computer Netwoks (IJCN), 2 (3), 152-158. 